\title{
Health Care and Hygiene Practices of Older People in Tea Garden: A Study Conducted in Lackatoorah Tea Garden of Sylhet District
}

\author{
Jiban Kumar Pal, Muhammed Muazzam Hussain* \\ Department of Social Work, Shahjalal University of Science \& Technology (SUST), Sylhet, Bangladesh \\ Email: jibanpal42@gmail.com, *muazzam777@yahoo.com
}

Received 25 April 2016; accepted 20 May 2016; published 23 May 2016

Copyright (C) 2016 by authors and Scientific Research Publishing Inc.

This work is licensed under the Creative Commons Attribution International License (CC BY).

http://creativecommons.org/licenses/by/4.0/

(c) (i) Open Access

\section{Abstract}

The recent changes in demographic structure cause a gradual increase in high proportion of older people in Bangladesh. Health status of the older people is a major concern in Bangladesh. Bangladesh lacks medical facilities and infrastructure to meet the needs of the vast tea garden population not to speak of the older population. The majority of tea garden older people in Bangladesh live in absolute poverty. As a result they deprive health care facilities and other old age services from family and society. The present study was conducted to explore the health care and hygiene practices of older people in tea garden areas and to find out their actual health situation, treatment experiences, health seeking behavior, hygiene, social attitudes towards them, social supports and their valuable recommendation for improving their present conditions. The present study was qualitative in nature. It was carried out on eleven villages (para/plot) at Lackatoorah tea garden area. The data for the study were collected from 20 older people and two focus group discussion with eight older people in each group having age 60 years and above and living in tea garden areas in Sylhet district. Purposive sampling technique was employed for selecting sample from the older people who live in the study areas. In the tea garden areas older people mostly suffer from old age related and communicable diseases due to unhygienic environment and practices. Lack of proper information and awareness and bad experiences in government hospitals, many extremely poor tea garden older people first sought treatment from cheaper or free traditional healers. The poor hygiene situation of tea garden areas in Sylhet district is mainly responsible for present health status and hygiene of older people. In tea garden areas older people lead a troublesome life due to insufficient income and allowances facilities.

\section{Keywords}

Older People, Tea Garden Areas, Sylhet District, Health Care, Hygiene, Social Attitudes,

"Corresponding author.

How to cite this paper: Pal, J.K. and Hussain, M.M. (2016) Health Care and Hygiene Practices of Older People in Tea Garden: A Study Conducted in Lackatoorah Tea Garden of Sylhet District. Open Journal of Social Sciences, 4, 144-154. 


\section{Social Support}

\section{Introduction}

Old age is a common phenomenon across the world and over time. It includes the proportion of older population aged sixty years and above. It is an emerging issue in Bangladesh. With the improvement in health care and increased life expectancy it is becoming a vital problem in Bangladesh. Over the last one decade, in Bangladesh the proportion of older population has been significantly increased. In Bangladesh, population ageing is viewed as a natural outcome of demographic transition from high fertility and mortality to low fertility and mortality due to the years of successful family planning (FP) and public health programs that have changed the population growth of the country [1]. The developed countries evolved policy instruments in the form of social security as well as care for the older people through institutions like old age homes, geriatric hospitals, old age recreation center and many other public and private care systems for the aged but in Bangladesh these facilities are not sufficient for older people specially for the tea garden older people. Bangladesh, as a developing country, has a long cultural tradition of looking after the older and families and communities are expected to take care of their own older members. Most of the older people in the country are suffering from many basic human problems such as lack of sufficient income and employment opportunities, absolute poverty, senile diseases and absence of proper health and medical facilities, exclusion, negligence, deprivation, socio-economic insecurity, etc. Health is one of the important human rights that strengthens the persistence of human being and help them to increase basic capabilities. The breakdown of traditional family pattern, changes in traditional norms and values, innovation of modern medical facilities and increasing the rate of older people. For these changes, the older people face difficulty to meet their physiological, psychological and social needs. They are mostly vulnerable in terms of living arrangement, food consumption and possessions on wealth, participation in decision making of the family and social attitude and values [2]. In Bangladeshi context because of traditional norms and religious and social values most of the older people are living with their offspring but at the same time it should be elicited that how easier this co-residency. Because of wide spread poverty and socio-economic change, living together is no guarantee of economic well-being of the older people [3]. Old age brings both physical ailments and social problems. Major social problem of the old people is their adjustment to their surrounding social world in general and their immediate families in particular. Old people very often feel neglected and forgotten. This gives rise to anger, sorrows and frustration leading to tension in the family [4]. In low-income societies, very few older people have access to any comprehensive care, and there is little or no emphasis on the importance of understanding the contextual reality of the older people's life. Social support for the older people mostly comes from the informal networks, often with a little support from semiformal network. Most of the older people receive little or no support from the formal network [5]. The tea garden older people avoid seeking health care from a formally qualified doctor due to high costs. Familiarity and accessibility of health care providers play important roles in health-seeking behavior of older people in tea garden areas. Flexibility of health care providers in receiving payment is a crucial deciding factor of whether or not to seek treatment, and even the type of treatment sought. Most significantly, old age is found to be an accepted explanation of ill health and the decisions taken to seek health care are influenced by factors such as perception of severity of illness, familiarity and accessibility to health care providers, and financing of health care. In the absence of specialized knowledge in geriatric health care, multiple sources of health care, such as allopathic care, kabiraji and homeopathic care are sought by the elderly people as also reported in an earlier survey on health-seeking behaviour of adults in rural Bangladesh. It is very important to understand the health needs of the older people and so solicit their opinion in improving the existing health care and hygiene system in the country. Therefore, this study was undertaken to understand the health care and hygiene practices of older people and to gather some information about their perceive health needs using the information and over of Lackatoorah tea garden in Sylhet district.

\section{Objectives of the Study}

The main objective of this study was to delineate about the health care and hygiene practices of older people in the tea garden areas of Sylhet District as well as to identify how the cultural patterns of tea garden areas affect the care of the older people. Therefore, the specific objectives were selected: 
- To explore their present health care and hygiene practices.

- To get a better understanding about their health seeking behavior.

- To identify the potential problems and challenges they face in getting services.

- To know about the familial and societal attitudes toward older people in tea garden areas.

- To present some recommendations to promote their health conditions and wellbeing.

\section{Conceptual Definitions of the Study}

For the purposes of the study, the following operational definitions of the key terms were used. Old age is considered the closing period of human life of an individual. The Constitution of Bangladesh in its clause 15 (d) clearly declares to introduce the Social Security Programme in 1998 for the older people who is 65 years or above for man and 62 years or above for woman [6]. The government, through the Public Servants (Retirement) (Second Amendments) Ordinance, 2012, promulgated on Dec 6, 2012, increased the retirement age of the public servants to 59 years from 57 years and the freedom fighter public servant will enjoy one more year in job before going on retirement [7]. Therefore the inclusive criteria were to become Bangladeshi citizens living in the tea garden areas in Sylhet District and having the age of sixty years and above. The area around Sylhet is a traditional tea growing area. In the tea garden area, most of the land is hill slopes and tea garden worker and their family live in hilly place. They have no their own land for building their houses. So, they live in small and soil made houses. In this study tea garden refers to a place where disadvantage indigineous people live with poverty, poor healths and nutritions, densely, inadequate lighting, lack of safe drinking water, water logging during rains, absence of toilet facilities and non-availability of basic physical and social services. Particularly older people suffer more in tea garden areas. Health care is generally used to indicate the diagnosis, treatment, and prevention of disease, illness, injury, and other physical and mental impairments in humans. In this study health care was used to refer to the government health care services, NGO, voluntary and family health care related services etc. In this study Sylhet district was used to refer the area of Sylhet district including the all tea garden in this region. Health seeking behavior was used to refer the way of seeking health care and their attitudes about health care. Hygiene is a set of practices performed for the preservation of health. Hygiene refers to conditions and practices that help to maintain health and prevent the spread of diseases. Social support was used to refer the services and allowances for older people and their health care and it was statutory and non-statutory support. The term attitude was used to refer the treatment behavior, thinking pattern and outlook of family members and community people towards older people in tea garden areas.

\section{Methodology of the Study}

This study was based on qualitative research method with various data collection procedures, such as interview, focus group discussion (FGD) were employed to collect necessary information. Although the study was based on primary data, a number of secondary sources, such as policy documents, journal articles, academic books, official documents were used to conduct the study. In this study Lackatoorah tea garden was selected for collecting information about health care and hygiene practices of older people. This tea garden has eleven villages (known as para/plot) and all tea garden workers live in that area with their older people. The older people live in soil made small houses with their other family members. The researchers were selected those villages purposely because Lackatoorah tea garden was near to researchers resident and had proper knowledge about the study area. The primary population of the study was comprised the aggregate number of older people living in Lackatoorah tea garden area of Sylhet District who belong to the age of sixty and above. The total older population of the above eleven villages were 108 and among them 67 female and 41 male person. Purposive sampling procedure was used to access the data necessary to address the research objectives. A number of 20 samples were purposively selected and collected information from each of them. In addition two focus group (FG) were formed with eight participants in each group. The methods/techniques of data collection were semi-structured interview schedule and focus group discussion (FGD). Apart from primary sources of data secondary source was used in order to understand the present situation of older people in slum areas.

\section{Validity and Reliability of Data}

Validity in research is concerned with the accuracy and truthfulness of scientific findings [8]. In this study, it was demonstrated what actually exists and a valid instrument or measure actually measured and what it was 
supposed to measure. Reliability is concerned with the consistency stability and repeatability of the informant's accounts as well as the investigators' ability to collect and record information accurately [9]. In this study the consistently of the same results over repeated testing periods was checked. During study period, the researcher using the same or comparable methods obtained the same or comparable results every time and used the methods on the same or comparable subjects. The researcher developed consistent responses or habit. The researcher collected information from the respondents through in-depth interview and focus group discussion and observed their present health conditions personally. Whenever needed, responses were cross-checked with the respondents over telephone. In case of content analysis, principle of authenticity and subjectivity was maintained. So data were credible and reliable as possible.

\section{Data Analysis}

Qualitative analysis of data requires an interpretive approach concerned with understanding the meaning which people attach to phenomena (actions, decisions, beliefs, values, etc.) within their social world [10]. The recorded interviews and focus group discussions were transcribed in full and the accuracy was checked against the original recording and noted by the researcher. The transcripts were analyzed using thematic analysis, derived deductively from the research questions and inductively from meanings or actual phrases used by the participants. The trustworthiness of the data analysis process was enhanced by the researcher. Triangulation of data was ensured by comparing focus group discussions and interviews data for identifying consistency and contradictions and eventually inconsistencies were examined and interpreted. In contrast, semi-structured interviews provided a great deal of information concerning personal issues. Interview and focus group discussion participants talked about their health and hygiene status, hospital experiences and the issues of general wellbeing. They were confident to talk about family matters including personal experiences of care, evaluation of self, and attitude towards diseases, treatment and future planning.

\section{Ethical Issues of the Study}

When we talk about ethics in social research we are addressing those issues that concern the behavior of social researchers and the consequences that their research brings to the people they study. As such ethical issues have the potential to impact at every stage of the research process and within any research project. This is particularly important issue in qualitative research where there is significant potential for misleading people to be genuinely interested, using contacts to gain confidential information, betraying confidences, and consequentiality. Participation in the study was voluntary and relied on the ethical principal of consent. At the start of interview and focus group discussion, the study purpose and nature were explained. Although the written consent was not taken from the respondent but verbal consent was taken from them. Since the most of the participants were illiterate in Bengali and English; their presence at the interview or focus group was taken to be consent. It was further explained that while the anonymity of individual participants were preserved that their verbatim may be published, although action was taken to prevent them being identified.

\section{Limitations of the Study}

The study was carried out on health care and hygiene practices of older people at tea garden areas in Sylhet District to investigate the present health situation and care practices. The data for the study was collected from 20 people having age 60 pulse and living in Lackatoorah tea garden areas and using purposive sampling technique and semi-structure interview schedule during the month June to July 2015. Only two focus group discussion consisting with eight older people in each group in tea garden areas and their family care givers. As a qualitative study the sample size was small and that could not represent the whole older people in tea garden areas in Sylhet District. The findings are not generalizable beyond the study sample.

\section{Findings of the Study}

\subsection{Demographic Information of the Respondents}

The demographic information of the population living in the studied area is important to understand the health care and hygiene practices characteristics of older people in the tea garden area. From the study it was seen the average age of respondents in tea garden areas in Sylhet District was 70.25. In the tea garden areas most of the 
older people live with their son's and daughter family. It was revealed that the thirty six percent respondents live in joint family with their sons and sixty four percent of respondents live single family with one son or daughter. Education is the backbone of the nations. Education is considered as an important factor of the socioeconomic characteristics of the household. It reveals from the collected information that only fifteen percent respondents completed primary schooling and other dropped out due to poverty of their parents and lack of educational facilities in the tea garden areas. Most of the older people are unemployed and they spend their time in home. Although a few numbers of older people work in tea garden but their income is very law. Twenty three percent respondents earn one thousand taka per month through picking tea leaf and planting in the garden. Older people in tea garden areas suffer various diseases, so, for their treatment and livelihood they need money. Their expenditure is high in respect of their income. It is seen that monthly expenditures of the respondents are three to four thousand taka and eighty five percent respondents depend on their offspring for daily food and medicine. Bangladesh is a multi-religious country. Mainly four types of religious people live in this country but in the tea garden they are indigenous and mainly belong to sanatan/hindu religion. Marital Status of the respondents was shown that thirty three percent of the respondents are widow and widower. The high proportion of widowhood among the older women and who live with their son or daughter's family. Loneliness is a major problem of the older people when they lose one marital partner.

\subsection{Diseases, Treatment Experiences and Health Seeking Behavior of the Respondents}

This study revealed that majority of the tea garden older people suffers from one or more diseases. In the Lackatoorah tea garden areas older people mostly suffer from the headache, skin diseases, fever, cough and cold, gastric/ulcer, toothache, diarrhea, jaundice and dysentery, heart disease, hypertension, cancer, asthma, depression, mental stress, feelings of insecurity. In the tea garden areas older people suffer various diseases and expressed their view. One 68 years old female interviewed participant said about hypertention,

"Most of the time I suffer miserable pain in my shoulder and head when my blood pressure increase. I cannot tolerate this pain. Although I take medicine regularly for hypertension but sometimes it raise due to anxiety for my present health condition and poverty of our family".

One FG participant shared his view in the following way about heart problem:

"I have heart problem from two years. Before nine months first time I felt heart attack and was admitted in Osmani Hospital. When I attacked this problem I felt moving my head and I fall down the yard. From that attack I lost my one leg energy and cannot move now without the stick".

The study data also describe that most of the older people in Lackatoorah tea garden use tobacco and alcohol and due to this they face some cancer diseases. One interview 63 years older male participant said,

"Regularly I use tobacco (gul) in my mouth and it has affected my mouth and teeth. I feel pain due to infection in my mouth. Although I have gotten treatment from Kabiraj but it has not worked properly. Beside this I take cigarette daily and it has created lung cancer and heart disease. Always I suffer cough and bleeding cough from mouth. I take medicine for TB and it damages my body".

Asthma is caused by a combination of complex and incompletely understood environmental and genetic interactions. From the collected data it was seen most of the respondents suffer asthma and problem in breath. One 78 years old FG participant included,

"About ten years I suffer asthma and it gives pain all time. I cannot take breath properly without inhaler and medicine. I know this disease has come from my father who had asthma. Another factor is dirty environment in our colony. In winter session I suffer much due to dust and cold wind. I went to hospital for treatment but said it will not be removed properly".

In the tea garden areas older people suffer from communicable diseases due to unsafe water and unclean environment, like skin diseases, fever, cough and cold, diarrhea, jaundice and dysentery. The study also shows that these common diseases attract older people. One of the focus group participants mentioned,

"In our tea garden, most of the people suffer diarrhea and dysentery for unclean water. I have dysentery problem and frequently I suffer this due to water and unclean environment and problem of damaged latrine. 
Last time, it was a miserable for me because as long ten days it was not cured".

The findings revealed that about all older people suffered skin diseases one or many times. Skin Diseases is common among the tea garden older people. One 63 years old female participant shared her view in the following way:

"I have been suffering khuja and gha (skin diseases) in my foot figure two months. It increases in night and then I feel miserable pain in my leg. Although I use antiseptics cream but it does not remove."

Treatment and health care is essential for older people. Most of the tea garden older people take treatment from M.A.G. Osmani Medical College Hospital and Ragib-Rabeya Hospital Sylhet. Most of the tea garden older people go to quack doctor or kabiraj for diseases. They have insufficient money for proper treatment, so, they cannot take proper medicine for remedy. The mobility and accessibility of older people are also critical. Especially for older women, a cultural stigma is attached to visiting a male doctor who is not directly a family member. Most of the time they visit pharmacy and quack doctor. One 71 years male respondent shared his opinion,

"When we suffer any diseases we go to pharmacy/village doctor for medicine and treatment. I like him (village doctor) because he is flexible with us. If we pay small amount he accept it. He understands poor people's problems and also gives good medicine. We always go to him and he is our regular doctor (village doctor). He is available whenever we need him, day or night he also comes to our home when needed. We are happy with him".

Due to these limitations on access and affordability, conditions meant that they were more likely to become ill, many visited other, less effective, service providers. Tea garden situated on public land also usually lacked public services such as health, water supply, sanitation, electricity and cooking gas facilities. Although some NGOs provide some health care services but these are not sufficient and proper for those older people in tea garden areas.

Because more effective sources of treatment were more expensive, and because of bad experiences in government hospitals, many extremely poor tea garden older people first sought treatment from cheaper or free traditional healers (kabiraj). Traditional healers were most commonly approached in cases related to dysentery, diarrhoea, fever, cough, typhoid, and other skin diseases. Seeking support from traditional healers like kabiraj or religious persons (Imam) through enchanted water (pani pora) or an amulet (tabeej), were thought to be best option to overcome diseases caused by evil spirits. Such interventions were damaging not only because they were ineffective, and sometimes directly harmful, but also because their use delayed treatment from proper health services.

The interview data also demonstrate that most of the participants face major troubles when they suffer diseases and fell illness and go to government hospital such as Osmani Hospital for treatment. A number of focus group participants discussed about diseases and treatment they receive from hospital and kabiraj or other quack doctor for their treatment in old age. One participant included,

"Most of the time I suffer dysentery, diarrhoea, fever, cough, and other skin diseases and my wife go Pharmacy and Kabiraj for medicine and pani para. I cannot go hospital for my energy lose and eye problem and insufficient money for treatment. In slums area these types of diseases are common and most of the people go Kabiraj and Imam. But I received an operation in my eye from Osmani Hospital free of cost”.

\subsection{Hygiene Situations of the Respondents}

\subsubsection{Hygiene Status}

The research findings suggest that older people hygiene is an issue that many caregivers have to take care them in tea garden areas. The FG data demonstrates that the some participants refuse to take a shower or bathe, change their clothes, brush their teeth or clean body due to illness, all of which result in bad older people hygiene. However, for many tea garden older people depending on others to help them with hygiene. One FG participant shares his view in the following way:

"I know cleanness is important for healthy life but I can't take bathe due to my asthma and cough illness. My wife helps me for boiling water for my bathe and sometimes clean cloths".

Another 71 years male interview participant said about, 
"I can't take bath daily due to my diseases and most of the time I wash my hand and leg. When want to take bath then my grandson help me for pumping the tubwell".

The study findings demonstrates that home hygiene practices prevent or minimize disease and the spreading of disease in home (domestic) and in everyday life settings. In the tea garden areas most of the participants are illiterate and have no sufficient knowledge about their home and environmental hygiene. One 74 years female participant included:

"I can't understand why people throw their garbage and food waste in road side. These waste rotten and bad smell spread over air. I try to clean my house but my neighbors are not aware about cleanness their house. Sometimes my daughter-in-law does not wash hand before serving food".

Another FG participant included,

"Although we live in unclean and dirty environment but it can be changed through our awareness. All time I say to my daughter-in-law for cleaning our house but she does not give importance for me. All time she and other people throw their household waste in road and outside of home".

\subsubsection{Sanitation Status}

Tea garden older people are marginalized and disadvantage. These people have limited knowledge about water, sanitation and hygiene. In the tea garden areas the people use unsafe and damage latrine with their neighbors jointly. One 70 years old female participant shares her experience:

"I face problem due to damage latrine because I feel insecurity for falling in waste when I use it. In this area the number of latrine is small with a view to huge people. Our latrine use five families, so we wait for using toilet when anybody uses it, but nobody repair it. This damage latrine creates bad smell and spread with air".

Another FG participant expressed his view,

"In our tea garden, we face many problems for shortage number of latrine and these are not good for using. All time these are busy and nobody clean it. When we need to use latrine then we wait for free of it. All latrines in our community are made with bamboo and slab. The sanitation system is not good and all time waste storage in commode".

The research findings suggest that in the tea garden areas most of the respondents have not own latrine and other participants use latrine jointly. They are not properly aware about their sanitation facilities. Cleaning of toilets and hand wash facilities are not proper. It is important to prevent odors and make them socially acceptable. Social acceptance is an important part of encouraging people to use toilets and wash their hands. One 74 years male interview participant said,

"I try to wash my hand after toilet but lack of soap I can't wash properly. When I take food then I wash my hand and mouth. In our tea garden most of the people are not aware about cleanness and hygiene. They take food without washing their hand".

Another FG participant expressed his view,

"In our community, only one time a campaign held for creating awareness about health, hygiene and nutrition. Most of the people know this but nobody follow these. In my house, I try to wash my hand after toilet and before taking food and I say my other family members for washing hand".

\subsubsection{Food Habits and Nutritional Status}

The study demonstrates that tea garden older people mostly eat rice, vegetables, and dull. Occasionally they eat fish, meat and milk. Most of the respondents suffer malnutrition due to insufficient income and resources.

Poverty is a strong predictor of poor health, and malnutrition is more prevalent among older persons who live in poverty. From the study it was seen that with low socio-economic status were found to suffer from more nutritional deficiencies. The consequences of malnutrition are diverse, severe and long-lasting. Malnutrition is associated with physiological, psychological, and immunological consequences and has a strong impact on mortality, morbidity, and quality of life. In addition, malnutrition increases vulnerability for infection, pressure sores, 
delayed wound healing, and reduces rates of drug metabolism.

The research findings suggest that the older people can't afford to take proper nutritious food due to lack of resources and low income of their and their sons in tea garden areas in Sylhet district. One 70 years male interview participant shares his experience as the following way:

"I can't not eat proper food for inability of my son who earns money through day plantation in the tea garden. The price of daily commodities is high and we can't afford to buy meat and fish daily. Most of the time I eat rice with dull and vegetables. Only three or four time we eat meat in a year, but it buy when guest visit to our house".

Another FG participant shared his view in the following way,

"Most of the time we take rice, dull, vegetables. We are not capable for eating rich food due to economic crisis. In our community, all people are poor so, they can't spend more money for older people for their nutritional food, medicine, treatment and others. I can't take proper nutritional food due to my family shortage resources".

\subsubsection{Water Situation}

Safe drinking water is essential to humans and other life forms even though it provides no calories or organic nutrients. From the study findings it was seen that tea garden worker have no own tubwell for drinking pure water. In the tea garden people get water from deep tubwell for pure drinking, cooking, bathing and other using water. Most of the tea garden older people are not aware about pure drinking water. They seem all tubwell water is pure. Pure drinking water crisis is high in tea garden areas. The people in tea garden areas quarrel with one another for collecting water and bathing. The older people are more vulnerable for bathing and collecting drinking water from tubwell because the floor of tubwells is slippery and unclean. One 70 years older female participant included:

"When my daughter-in-law is busy for other work then I go to collect drinking water from tubwell and it is difficult for me because it is busy all time and I can't compete with other for collecting water. Many times I have fallen on tubwell due to slip of floor. All tea garden people wash their cloth and utensils (dish/plate) on tubwell and throw food waste on it. These wastes create dirty outside the tubwell and create bad smell".

Another 76 years older male participant shared his view,

"Most of the times in our community people collect water from manager house because our community's tubwell is not work properly and it was damaged before three month. Nobody repair this tubwell. Sometimes I take bath on tubwell but it is slippery and many times I have fallen down."

The study demonstrated that in the garden areas most of the older people as well as all people face problem due to limited supply of pure drinking water. One 70 years old female interview participant said,

"In our community, we can't get sufficient pure drinking due to huge population. In this tea garden two tubwell is active and other is out of work. So, all time we wait for collecting drinking water. Most of the time the community people qurral each other for collecting water and taking bath. In the morning, we face huge problem for collecting water because that time all community people gather tubwell for collecting water, bathing, cleaning utensils. In our tea garden we don't get the supply water, so, we all time depend on only these two tubwell for water".

\subsection{Societal and Familial Attitude towards Respondents}

Community people of tea garden areas are not literate and they have no proper knowledge about health care practices of older people. These tea garden areas people preserve mostly negative attitude towards older people because they do not work and earn money. One participant shares his view like

"My family members help me about my daily activities and take care properly. Sometimes my son's wife angry with me due to my illness and old age. My son takes me hospital when I am sick and need treatment".

Another 67 years older female participant shared her experiences, 
"Although community people respect me but my daughter-in-law can't take proper care due to her work. I always stay home and gossip with my neighbor and take care my grandsons".

Family is the place where old people can get proper care and support for living comfortably. Different people in society are not sensitive to the older people. They make various comments towards older people in modern age. In the tea garden areas most people living with poverty; so, they cannot behave positively with their older family member. One respondent said.

“Sometimes son's wife neglect about myself diseases but most of the time my son take care me properly".

The attitude toward older people is changing and a number of participants also shared rational attitudes towards them and family member and society people respect older people now. One 78 years female respondent included,

"Most of the society people had negative attitude towards older people because they suffer various diseases and unable to do something. Although this situation is changing but sometimes family member anger with me for my diseases".

The study demonstrated that older people have a feeling of being neglected by the society because nobody has the time to sit with them. Generation gap increases this situation and increases the feeling of despondency. In old age, they felt they had become less active and due to their poor economic condition, they can't attend the social gatherings. One 72 years older female interview participant shared her view in the following way,

"I can't understand why I don't get the company of others. All time I stay home alone and feel loneliness. My son goes outside home for work and daughter-in-law is busy for familial work. Most of the time I feel nobody take care me and they have no time for sitting with me".

\subsection{Social Supports and Informal Care}

The Universal Declaration of Human Rights 1948, recognized health care as one of the fundamental rights for every human being. Social support is needed for an older people for leading his/her life. These support or services may be statutory and non- statutory. Each country introduces various services for his vulnerable and disadvantage people. Older people are considered most senior and vulnerable. In tea garden areas older people lead a troublesome life due to income and money or allowances from state. One respondent says

"Many people get allowance but I do not get these, how I will get old age allowance and when. I got some support from my neighbour."

Poorer families face yet more liabilities and very often resign themselves to their ill fate. At this point they do not seek any health care services and are forced to cope with increasing discomfort. If the older person gets better at this point, they try to recover the financial losses and liabilities over a period of time. If the initial treatment does not work and the older patients are referred or advised to seek specialized treatment. Sometimes even the neighbours help with some money, one respondent said,

"My sons are poor and they have their own families to take care of, so they can't help me but my neighbours helped me and gave me some money for treatment."

Another 71 years older male participant shared in the following way,

"Although I did not get any allowance from government but sometimes I get support from my daughter and neighbors. All time my neighbors help me for visiting doctor and buying medicine."

Many NGOs run various programs and services for the tea garden people about their health and nutrition, hygiene, sanitation etc. The study reveals that these types of services help the older people who live in tea garden areas. One participant said,

"I do not get any government support. Before two years I visited ward commissioner house for getting any allowance but he said, I can get next year but, I did not get anything. Only one time I got free medical treatment from NGO."

The study revealed that majority of the older people in tea garden areas prefer to get care from family members 
because they feel comfortable in family and family members are well known about their conditions. The family members, relatives, friends and neighbors provide the bulk of the support of the older people who require assistance. Such informal support is more cost effective and satisfying to the older people than the public support systems, which are often not accessible to the poor. One FG female participant shared her

"In our tea garden we are mostly familiar with one another and we need help then we try to help one another. Previous year when I felt headache and pain in my chest and shoulder then next door neighbor took me hospital as soon as possible. If he did not take me then I would die. When I need money for medicine then my son and daughter help me as possible as their affords."

The traditional norms and values of Bangladeshi society stress respect and provision of care for the older people, however, the ongoing processes of urbanization, industrialization, modernization, globalization and their concomitant processes have led to changes in the traditional support base of the older people. The study was also shown that most of the tea garden older people live with their offspring.

\section{Recommendations}

The existing services are quietly inadequate and insufficient. Older people are increasing in an alarming rate day by day. Insufficient and disorganization in the health care services, social prejudice and beliefs, social structure and system, negative attitude towards older people are also intensifying their problems. The health care and hygiene practices of older people in tea garden areas are at the risk of being the poorest of the poor. In order to make easier the life of the older people in tea garden areas in Bangladesh some initiatives should be taken.

According to the findings of the study, the recommendations that may be put forward in this regard are as follows:

1. The Department of Health should establish mini public clinics within the tea garden or adjacent areas especially targeting the older people which can act as a branch/referral centres to connecting public hospitals;

2. A friendly information desks can be established at public hospitals especially for older people who have limited knowledge and capacity to get access to services;

3. Micro-health insurance for disadvantages poor older people, and especially the extreme poor, may be one of the better options towards ensuring the adequate health care.

4. Government should take some programs for making them involvement through using their inner potentialities and experiences to reduce their mental suffering resulting from loneliness and isolation.

5. The amount of old age allowance and beneficiaries should be increased at satisfactory level.

6 . The government has to take necessary actions to the problem of poor housing, water and sanitation facilities in the tea garden areas.

7. Various NGOs engaged in tea garden development activities need to take steps for wider literacy and health care programme in order to accelerate improvement in annual income.

8. Our electric media can take an action to increase public awareness and health care and hygiene practices of older people in tea garden areas.

\section{Conclusions}

The tea garden workers have indigent socio-economic condition especially their level of education and income is inadequate. Consequently, they are not conscious of as well as unable to get the health services for older people after meeting their everyday needs. The study shows that near about all of the respondents live in one room houses which have no windows and most of the floors as well as roofs of the houses are made of mud and tin with cane respectively. As most of the people live hand to mouth, their living condition is not up to the standard at all. These respondents are economically poor and they depend on their offspring for treatment and other health care and sometimes it may prevent them from taking treatment. With regard to the health care of the respondents, it was found that the majority respondents had reported with one or another type of disease and sickness, hence the circumstances reveals that old age is associated with disease and illness. The attitude toward older people is changing and a number of participants also shared rational attitudes towards them and family member and society people respect older people now. Social support is needed for an older people for leading his/her life. These support or services may be statutory and non-statutory. Each country introduces various services for his vulnerable and disadvantage people. 
The study shows that sanitation and hygiene condition is worse as most of the respondents said that they use open latrine and do not wash their hand by ash or soap after defecating or before eating. Similarly they also informed that they are not aware of the sources of water. As a result they use lake (known as sora) and supply water rather than boiled drinking water. However, the condition of the place of water supply is not clean. A few number of small slab stand in an open place, which is widely used for the purpose of bathing and daily domestic work. Tea garden older people are not aware about their health care, hygiene and sanitation practices. So, a universal agreement is essential among the policy makers regarding the tea garden older people for the health care services, basic rights, social security and psychological status. The media should assist by presenting positive images of tea garden older people, particularly emphasizing the need for respect because of their past and continuing contributions to society.

\section{Acknowledgements}

Financial support from Department of Social Work, Shahjalal University of Science and Technology, Sylhet, Bangladesh is gratefully acknowledged. The family member of Lackatoorah Tea Garden of Sylhet District, Sylhet, Bangladesh is gratefully acknowledged for their kind cooperation regarding the provided their important and relevant information to complete this research work successfully. Ms. Amina Pervin, Professor, Department of Social Work, Shahjalal University of Science \& Tecnology, Sylhet, is gratefully acknowledged for her kind cooperation and mental support.

\section{References}

[1] Strong, M.A. (1992) The Health of Adults in Developing World: The View from Bangladesh, Forum: Adult Mortality. World Bank Publication, Washington DC.

[2] Hossain, I., Akhtar, T. and Uddin, T. (2006) The Elderly Care Services and their Current Situation in Bangladesh: An Understanding from Theoretical Perspective. Journal of Medical Sciences, 6, 131-138.

http://dx.doi.org/10.3923/jms.2006.131.138

[3] Kabir, H. (1994) Local Level Policy Development to Deal with the Consequences of Population Aging in Bangladesh. United Nations, New York, 33.

[4] Amirun, N.M, Haque, M.E., Siddiqua, N.M.R. and Haque, M.I. (2013) Social Status of Elderly People in Health Perspective: A Comparison of Rural and Urban Area. OSR Journal of Humanities and Social Science (IOSR-JHSS), 18, 83-94.

[5] Rahman, M.M. (2009) Health Status and Health Needs among the Aged Population in Chapai Nawabganj District of Bangladesh. Indian Journal of Gerontology, 23, 32-41.

[6] The Constitution of the People’s Republic of Bangladesh. http://bdlaws.minlaw.gov.bd/pdf_part.php?id=367/

[7] The Public Servants (Retirement) (Amendment) Act 2013. http://www.dpp.gov.bd/upload_file/gazettes/9818_55468.pdf

[8] Le Comple, M.D. and Goetz, J.P. (1982) Problems of Reliability and Validity in Ethnographic Research. Review of Educational Research, 52, 31-60. http://dx.doi.org/10.3102/00346543052001031

[9] Seltiz, C., Wrightsman, L.C. and Cook, W.S. (1976) Research Methods in Social Relations. 3rd Edition, Holt Rinehart \& Winston, New York.

[10] Denzin, N.K. and Lincoln, Y.S. (2003) Handbook of Qualitative Research. 2nd Edition, Sage, Thousand Oaks, CA. 